\title{
Kappeler, Manfred
}

\section{Sprechen über sexuelle Gewalt in pädagogischen Settings}

\author{
Erziehungswissenschaft 28 (2017) 54, S. 51-61
}

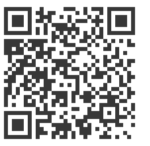

Quellenangabe/ Reference:

Kappeler, Manfred: Sprechen über sexuelle Gewalt in pädagogischen Settings - In:

Erziehungswissenschaft 28 (2017) 54, S. 51-61 - URN: urn:nbn:de:0111-pedocs-148778 - DOI: 10.25656/01:14877

https://nbn-resolving.org/urn:nbn:de:0111-pedocs-148778

https://doi.org/10.25656/01:14877

in Kooperation mit / in cooperation with:

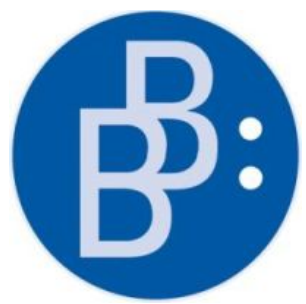

https://www.budrich.de

\section{Nutzungsbedingungen}

Gewährt wird ein nicht exklusives, nicht übertragbares, persönliches und beschränktes Recht auf Nutzung dieses Dokuments. Dieses Dokument ist ausschließlich für den persönlichen, nicht-kommerziellen Gebrauch bestimmt. Die Nutzung stellt keine Übertragung des Eigentumsrechts an diesem Dokument dar und gilt vorbehaltlich der folgenden Einschränkungen: Auf sämtlichen Kopien dieses Dokuments müssen alle Urheberrechtshinweise und sonstigen Hinweise auf gesetzlichen Schutz beibehalten werden. Sie dürfen dieses Dokument nicht in irgendeiner Weise abändern, noch dürfen Sie dieses Dokument für öffentliche oder kommerzielle Zwecke vervielfältigen, öffentlich ausstellen, aufführen, vertreiben oder anderweitig nutzen

Mit der Verwendung dieses Dokuments erkennen Sie die Nutzungsbedingungen an.

\section{Terms of use}

We grant a non-exclusive, non-transferable, individual and limited right to using this document.

This document is solely intended for your personal, non-commercial use. Use of this document does not include any transfer of property rights and it is conditional to the following limitations: All of the copies of this documents must retain all copyright information and other information regarding legal protection. You are not allowed to alter this document in any way, to copy it for public or commercial purposes, to exhibit the document in public, to perform, distribute or otherwise use the document in public.

By using this particular document, you accept the above-stated conditions of use.

\section{Kontakt / Contact:}

\section{peDOCS}

DIPF | Leibniz-Institut für Bildungsforschung und Bildungsinformation Informationszentrum (IZ) Bildung

E-Mail: pedocs@dipf.de

Internet: www.pedocs.de

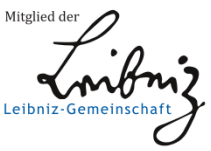




\section{Erziehungswissenschaft}

Mitteilungen der Deutschen Gesellschaft für Erziehungswissenschaft (DGfE)

Heft 54

28. Jahrgang 2017

ISSN 0938-5363

Verlag Barbara Budrich 


\section{INHALTSVERZEICHNIS}

EDITORIAL

BEITRÄGE ZUM THEMA

,ZUR ROLLE DER ERZIEHUNGSWISSENSCHAFT IM RAHMEN

DER DEBATTE UM SEXUELLE GEWALT IN PÄDAGOGISCHEN

KONTEXTEN“

\section{Fabian Kessl}

Die Erziehungswissenschaft und ihre ,pädagogischen Täter“.

Eine kommentierende Einordnung des Themenschwerpunktes

Jürgen Oelkers

Warum hat niemand den Verdacht geteilt? Die Odenwaldschule,

die Medien und die Erziehungswissenschaft.

Dieter Nittel

Gerold Becker: Lizenz zum sexuellen Missbrauch.

Ein Kommentar zu Jürgen Oelkers’ Buch „Pädagogik, Elite,

Missbrauch“ (Weinheim 2016) aus der Sicht der pädagogischen

Berufsgruppenforschung

Meike Sophia Baader

Zwischen Enttabuisierung und Entgrenzung.

Der Diskurs um Pädosexualität und die Erziehungs-, Sexual- und

Sozialwissenschaften der 1970er bis 1990er Jahre

Sabine Andresen \& Marie Demant

Worin liegt die Verantwortung der Erziehungswissenschaft?

Ein Diskussionsbeitrag zur Aufarbeitung sexualisierter Gewalt in der

Erziehungswissenschaft

Manfred Kappeler

Sprechen über sexuelle Gewalt in pädagogischen Settings

Alma Elezovic, Ingrid Lippitz \& Ulrike Loch

Heilpädagogische Diagnostik.

Zur wissenschaftlichen Stigmatisierung von Kindern und Jugendlichen, die an den Folgen von sexualisierter Gewalt litten ....

Jens Brachmann

Pädosexuelle Gewaltverbrechen - Erwartungen an die „wissenschaftliche“ Aufarbeitung 


\section{AllgEMEINE BEITRÄGE}

Ludwig Huber \& Karin Reiber

Hochschule und Hochschuldidaktik im Blick der

Erziehungswissenschaft

\section{MitTEILUNGEN DES VORSTANDS}

Stellungnahme des Vorstands der Deutschen Gesellschaft für

Erziehungswissenschaft (DGfE) zur Diskussion um sexuelle Gewalt in pädagogischen Kontexten

Stellungnahme zum Beschluss des Vorstandes der DGfE,

Hartmut von Hentig den Ernst-Christian-Trapp-Preis abzuerkennen

Ludwig Huber \& Klaus Jürgen Tillmann

Zur Erläuterung der Stellungnahme zur Aberkennung des Ernst-Christian-Trapp-Preises für Hartmut von Hentig

Inklusion: Bedeutung und Aufgabe für die Erziehungswissenschaft.

Stellungnahme der Deutschen Gesellschaft für Erziehungswissenschaft (DGfE)

Bericht über das Auswahlverfahren von Beiträgen für den 25. DGfEKongress „Räume für Bildung. Räume der Bildung “ in Kassel 2016.

Auszug aus dem Protokoll der 3. Vorstandssitzung

des DGfE-Vorstands der Periode 2016/2018

Fachtagung ,, Universität 4.0.' Folgen der Digitalisierung

akademischer Lehre und Forschung "der Deutschen Gesellschaft für

Erziehungswissenschaft (DGfE)

\section{BERICHTE AUS DEN SEKTIONEN}

Sektion 1 - Historische Bildungsforschung.....

Sektion 2 - Allgemeine Erziehungswissenschaft

Sektion 3 - Interkulturelle und International Vergleichende Erziehungswissenschaft (SIIVE)

Sektion 5 - Schulpädagogik

Sektion 7 - Berufs- und Wirtschaftspädagogik

Sektion 8 - Sozialpädagogik und Pädagogik der frühen Kindheit 


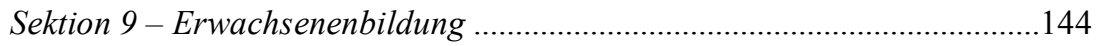

Sektion 10 - Pädagogische Freizeitforschung und Sportpädagogik .............146

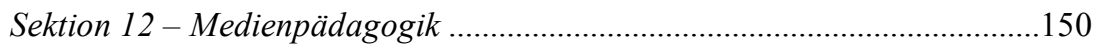

NOTIZEN

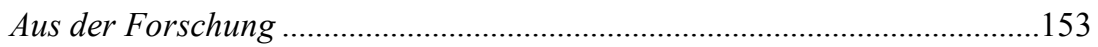

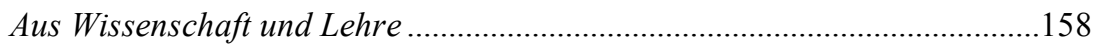

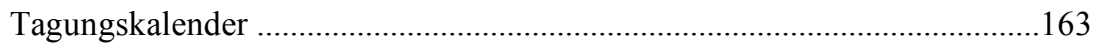

PERSONALIA

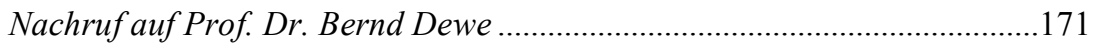

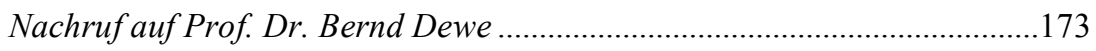

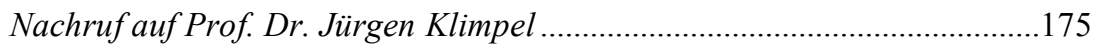




\title{
Sprechen über sexuelle Gewalt in pädagogischen Settings
}

\author{
Manfred Kappeler
}

Sexuelle Gewalt in pädagogischen Einrichtungen kann nicht allein aus einer Täterinnen- bzw. Täter-Opfer-Konstellation heraus erklärt werden. Sie entsteht und geschieht in der Binnenstruktur stationärer und ambulanter pädagogischer Settings (Heim, Internat, Kindertagesstätte, Jugendgruppe, Sportverein etc.).

Zur Binnenstruktur einer pädagogischen Einrichtung gehört auch die Sprache, in der die in der Einrichtung sich begegnenden Menschen, die erziehenden, pflegenden und versorgenden Erwachsene und die ihnen anvertrauten Kinder und Jugendlichen miteinander und untereinander über das Leben in ihr reden. Zu diesem Leben gehört die Sexualität. Für die Sprache, mit der sie im Binnenraum kommuniziert und nach außen in den gesellschaftlich geführten Diskurs eingebracht wird, gibt es eine Verantwortung des Trägers, der Leitung und der Mitarbeitenden, die ich in dem Begriff ,institutionelle Verantwortung" zusammenfasse.

Zum Sprechen über Sexualität gehört auch die Thematisierung sexueller Gewalt. Das Sprechen darüber fällt erziehenden Erwachsenen untereinander und im Gespräch mit Kindern und Jugendlichen noch schwerer, als das Sprechen über Sexualität generell. Die Kommunikation über Sexualität gibt Aufschluss darüber, wie in einer pädagogischen Einrichtung mit diesem komplexen Thema umgegangen wird. Sprache und Sprechen, Schweigen und Verschweigen, Hören und Zuhören bilden einen Zusammenhang, zu dem auch die Körpersprache sowie das Sehen, Hinschauen und Wegsehen gehören. Alle diese Handlungen gehören zur Kommunikation, die immer mehr ist als das wechselseitige Sprechen und Zuhören. ${ }^{1}$

In diesem Beitrag geht es aber hauptsächlich um die Sprache/das Sprechen. Es gibt keinen Aspekt der Sexualität, von der Artikulation des Sexuellen bis hin zur Befähigung von Kindern und Jugendlichen ,erlebtem Unrecht einen Namen geben zu können“ (Wolff 2015, S. 681), der nicht sprachlich vermittelt ist. Das gilt selbst noch für das bezogen auf Sexualität sehr häufige Schweigen und Verschweigen, denn auch das stumme Denken, unsere nicht ausgesprochenen Gedanken und Phantasien bedürfen der sprachlichen Form. Sie sind ein ,stummes“ Selbstgespräch.

In den folgenden Überlegungen wird es zunächst um das öffentliche Sprechen über sexuelle Gewalt gehen, den sogenannten Diskurs, und dann um die

1 Vgl. dazu ausführlicher: Widersprüche. Zeitschrift für sozialistische Politik im Bildungs-, Gesundheits- und Sozialbereich, 143, 2017: Sprache und Sprechen in der Sozialen Arbeit. 
Schwierigkeiten professioneller Erzieherinnen und Erziehern, in pädagogischen Einrichtungen mit den dort lebenden Kindern und Jugendlichen über Sexualität zu sprechen.

\section{Das öffentliche Sprechen über sexuelle Gewalt}

Die Professionellen auf allen Ebenen, von den Erzieherinnen und Erziehern in den Gruppen über die Leitungen der Einrichtungen bis hin zu den Verantwortlichen von Trägern und Dachorganisationen, sind mehr oder weniger am öffentlichen Diskurs über sexuelle Gewalt beteiligt. Für die Medien, die Politik, die Öffentlichkeit überhaupt sind sie sogenannte primäre Definierer: Die „die es ja wissen müssen“, weil sie doch die Expertinnen und Experten der Erziehungspraxis sind und den Alltag in den Einrichtungen genau kennen. Der öffentliche Diskurs über sexuelle Gewalt wirkt also nicht nur auf die Sichtweisen und die Sprache in den Einrichtungen/Settings zurück, er wird aus ihnen auch gespeist.

Für alle, am öffentlichen Sprechen über Sexualität und sexuelle Gewalt Beteiligte gilt, diese Beteiligung bewusst, unter Beachtung diskursethischer Anforderungen zu gestalten. Im Unterschied zur individuellen Ethik zielt die Diskursethik auf in einem intersubjektiven Prozess gefundene Problemlösungen ab (zum Beispiel der Verbesserung des Schutzes von Kindern/Jugendlichen vor sexueller Gewalt); Lösungen, die über den „Einzelfall“ hinausgehen. Ein Diskurs kann nur gelingen, wenn die an ihm mit Rede und Schrift Beteiligten wahrhaftig und verlässlich sind: Wort, Absicht und Handeln sollen übereinstimmen. Bei den in einen Diskurs eingebrachten Bezeichnungen und Beschreibungen einer Sache, eines Verhältnisses, eines Geschehens geht es nicht nur um die Semantik, sondern um die Antworten auf eine verstandene Situation, die den von ihr Betroffenen gerecht werden. Wenn ich am Diskurs teilnehme, bin ich mitverantwortlich für die diskursive Öffnung von Realisierungschancen für soziale und pädagogische Verbesserungen, in unserem Fall der Bedingungen für ein gelingendes Aufwachsen von Kindern/Jugendlichen in Einrichtungen der Kinder- und Jugendhilfe (vgl. Böhler/Gronke 2011).

Im öffentlichen Diskurs, aber auch im ,privaten“ Sprechen hat der Begriff sexueller Missbrauch, eine große Bedeutung. Mit ihm wird die Kindern und Jugendlichen von Erwachsenen zugefügte sexuelle Gewalt bezeichnet und beschrieben. Wer „Missbrauch“ sagt, setzt sprachlogisch „Gebrauch“ voraus, ohne ihn explizit zu thematisieren. Dieses Nicht-Erkennen der in der Rede vom „Missbrauch“ verborgen mitschwingenden Möglichkeit des „Gebrauchs“ kann ganz unterschiedliche Motive haben. Der Terminus „Alkoholmissbrauch" beschreibt einen problematischen, weil sozial- und gesundheitsschädlichen Alkoholkonsum. In unserer Gesellschaft dominiert aber die Auffassung, dass es einen kulturell und sozial integrierten und individuell verant- 
wortungsbewussten Gebrauch dieser Droge gibt, ja, dass ihr Gebrauch ihren Missbrauch bei Weitem überwiegt und es keinen Grund gibt, den Alkoholkonsum gesetzlich zu verbieten. Beim Begriff „sexueller Kindesmissbrauch“ verhält es sich genau umgekehrt: Einen ,sexuellen Gebrauch“ von Kindern durch Erwachsene, der sprachlogisch als Möglichkeit in ihm enthalten ist, darf es, in welcher Form auch immer, nicht geben. Bei sexuellen Handlungen Erwachsener an/mit Kindern handelt es sich immer um die Ausübung von psychischer und physischer Gewalt zur Erlangung sexueller Befriedigung des Erwachsenen, legiert mit der Befriedigung von Machtstreben, mithin um sexuelle Gewalt. Sie ist nach dem StGB eine schwere Straftat gegen die sexuelle Selbstbestimmung.

Es liegt nicht in unserem Belieben, Semantik und Grammatik der von uns benutzten/gewählten Worte zu verändern. Sie haben ihre gegenwärtigen komplexen Bedeutungen in einem langen historischen Prozess bekommen, die wir nicht durch einen definitorischen Willkürakt einfach aushebeln können. Wer also „Missbrauch“ sagt, setzt unweigerlich „Gebrauch“ voraus, auch wenn das ihrer bzw. seiner Intention widerspricht und ihr bzw. ihm das im Moment des Sprechaktes nicht bewusst ist. So sehr hat sich dieser „Sprachgebrauch“ in der Mediensprache und der von ihr stark beeinflussten, aber auch ihr vorausgehenden Alltagssprache festgesetzt, dass sie sogar in den Fachsprachen von diversen, am Diskurs über sexuelle Gewalt beteiligten Professionen wie selbstverständlich benutzt wird. Da ist von „Missbrauchsgeschehen“, von „Übergriffen“, ,sich vergreifen“, ,sich vergehen“ die Rede, manchmal sogar von „Ausrutschern“. Oft wird das sexuelle Gewalthandeln durch Formulierungen wie „das so etwas in unseren Einrichtungen geschehen konnte“ oder einfach nur „das“ und „so etwas“ ganz zum Verschwinden gebracht. Dieses Sprechen verwendet ein Vokabular der Beschwichtigung, Verharmlosung und Neutralisierung. Es ist ein euphemistisches Vokabular, mit dem Sätze gebildet werden, die man in Verbindung mit den Kontexten, in denen sie gesprochen werden, als „Missbrauchsjargon“ bezeichnen kann. Es handelt sich um eine das Schreckliche relativierende, es tendenziell entwirklichende Sprache, die es in Einem zwar auf verstellende und verkürzende Weise ,sagbar" macht, es aber gleichzeitig verdeckt. Sie bildet die Vorstufe zum Ver-Schweigen.

Kritikerinnen und Kritikern dieses Sprechens über sexuelle Gewalt wird entgegengehalten, es sei doch einerlei, wie dieses schreckliche Handeln genannt werde. Jeder wisse doch, was damit gemeint sei. Wichtig sei doch nur, dass jetzt endlich überhaupt darüber öffentlich gesprochen werde und nun auch die Opfer über ihre Erfahrungen sprechen könnten und gehört würden. Die so argumentieren, sehen nicht, dass der im „Missbrauchsjargon“ geführte öffentliche Diskurs die Möglichkeiten der Opfer, über ihre Gewalterfahrungen öffentlich und privat sprechen zu können, präformiert und begrenzt. Die Art und Weise, wie in der Öffentlichkeit über die Kindern/Jugendlichen angetane sexu- 
elle Gewalt geredet und wie in pädagogischen Settings über sie gesprochen wird (es gibt einen Unterschied zwischen Reden und Sprechen), ist nicht neutral und beliebig. Es gibt Gründe dafür, wie jeweils über sexuelle Gewalt gesprochen wird. Gründe, die eine (selbst)reflexive und (selbst)kritische Erziehungswissenschaft und Pädagogik/Sozialpädagogik aufklären muss.

Segmentierungen des Sprechens, die Ausblendung wichtiger Aspekte, ermöglichen die beschwichtigende Sortierbarkeit eines für das persönliche und berufliche Selbstverständnis (bezogen auf Sexualität gibt es da starke Korrespondenzen) bedrohlichen Themas. Die bei allen „Aufarbeitungen“ von im öffentlichen Bewusstsein lange Verdrängtem und Verschwiegenem zu beobachtende Tendenz, das Bedrohliche und Entsetzliche an ihm sprachlich zu verharmlosen, ist immer dann besonders ausgeprägt, wenn die ans Licht gebrachten Tatsachen geeignet sind, das von einer beteiligten Profession tradierte und gepflegte Selbstbewusstsein zu erschüttern (Soziale Arbeit als „helfender Beruf", Erziehung „vom Kinde aus"), oder wenn es um bedeutende gesellschaftliche Organisationen wie die Kirchen und andere Träger der Sozialen Arbeit bzw. Institutionen der Erziehung geht, die „Corporate Identity“ nachhaltig zu beschädigen, sie in der Öffentlichkeit zu diskreditieren. Der sprachliche Umgang mit der nach langem Verschweigen und Vertuschen offengelegten sexuellen Gewalt in pädagogischen Einrichtungen ist für diese Strategie ein beeindruckendes Beispiel. Zur Abwehr der von der sexuellen Gewalt an Kindern/Jugendlichen - konkret von ihren Praktiken und ihren Folgen für die Opfer - ausgehenden In-Frage-Stellungen von Professionen und Institutionen werden schadensbegrenzende bzw. -abwendende Sprachregelungen gefunden und benutzt. Sie zeugen nicht selten von Gleichgültigkeit oder gar Ignoranz gegenüber dem Leiden und Weiterleiden der Opfer und können bei ihnen zu (erneuter) Sprachlosigkeit und zu Re-Traumatisierungen führen.

Zum öffentlichen Diskurs über sexuelle Gewalt gehören auch die ritualisierten und formelhaften Äußerungen des „Bedauerns“ und der „Entschuldigung" von Verantwortlichen in Kirchen, Verbänden und Einrichtungen: „Sollte auch in unseren Einrichtungen Kindern und Jugendlichen Leid zugefügt worden sein, so bedauern wir das zutiefst ...". Betroffene verstehen dieses „Reden“ als Verweigerung der von ihnen erwarteten vorbehaltlosen Anerkennung der ihnen angetanen Gewalt und ihrer Folgen. Dieses Reden im Konjunktiv (,sollte“, „hätte“, „könnte“) hat einen Subtext der Verdächtigung, mit dem die von Opfern berichteten Gewalterfahrungen unterschwellig bezweifelt werden (vgl. dazu Kappeler 2015). Er resultiert aus einem reflexartigen Vorbehalt gegenüber den Menschen, die sich dazu durchgerungen haben, über die ihnen zugefügte sexuelle Gewalt öffentlich zu sprechen. Solche Redewendungen bzw. Sprachregelungen entwirklichen die erfahrene sexuelle Gewalt, weil sie nicht ihren Namen bekommt. Mit dem unspezifischen Begriff „Leid“, der jegliche Art menschlichen Leidens umfasst, wird das kon- 
krete Handeln, die Kindern von Erwachsenen zugefügte sexuelle Gewalt, neutralisiert und verdeckt.

Mit ihren formelhaften Äußerungen des „Bedauerns“ und „Entschuldigens" bestreiten und verdecken die Sprechenden (zum Beispiel sogenannte Missbrauchsbeauftragte der Kirchen) auch die Mit-Schuld der Institutionen. Diese Mitschuld liegt in der institutionellen Verantwortung für strukturelle Bedingungen einer Einrichtung/eines Settings, die sexuelle Gewalt ermöglichen, teilweise sogar fördern: die Auswahl, Qualifizierung und Weiterbildung des Personals, die Binnenorganisation der Lebensbedingungen der Kinder und Jugendlichen, fehlende Transparenz und Abschottungstendenzen gegenüber dem sozialen Umfeld und behördlichen Kontrollinstanzen, fehlende Beschwerdemöglichkeiten, Tabuisierung von Sexualität, hierarchische Leitungsstrukturen etc. (vgl. Kappeler 2011; Böllert/Wazlawik 2014). In den von den Sprechenden der Institutionen vorgetragenen Formeln des „Bedauerns" und „Entschuldigens" wird die von den Opfern erwartete institutionelle Verantwortung nicht übernommen.

An den Runden Tischen „Heimerziehung“ und „Sexueller Kindesmissbrauch" war die neutralisierende und entwirklichende Sprache der Politikerinnen und Politiker sowie Jugendhilfefunktionärinnen und Jugendhilfefunktionären für die in diesen Gremien beteiligten Opfer von sexueller Gewalt in Heimen und Internatsschulen eines der größten Probleme.

Es gibt drei Ebenen der Verantwortung für sexuelle Gewalt in pädagogischen Einrichtungen/Settings, die jede eine eigene, spezifische Verantwortung haben, die nicht gegen die der anderen aufgerechnet werden kann:

- die Verantwortung von in pädagogischen Einrichtungen/Settings arbeitenden oder sich um eine Anstellung in ihnen bewerbenden Männern und Frauen, deren sexuelles Begehren sich auf Kinder/Jugendliche richtet;

- die Verantwortung von Trägern und Einrichtungen für Strukturen, in denen sexuelle Gewalt entsteht und geschieht;

- die Verantwortung von Genehmigungs- und Aufsichtsbehörden, die für die Heimaufsicht und die Schulaufsicht gesetzlich zuständig sind (vgl. Kappeler 2014a, S. 15ff.).

Freilich ist der Missbrauchsjargon sehr oft auch „nur“ ein Ausdruck sprachlicher Hilflosigkeit bzw. der Unfähigkeit, sich von den herrschenden Sprachkonventionen zu befreien. Wie auch immer: Es handelt sich um eingefahrene Diskurse, die den Blick auf die Gewalt und ihre Opfer verstellen, ganz gleich, ob mit Kalkül betrieben oder aus Unwissen bzw. Hilflosigkeit im sprachlichen Mainstream mitredend. 


\section{Das Sprechen über Sexualität und sexuelle Gewalt in pädagogischen Einrichtungen und Settings}

Christiane Thompson schreibt in ihrem Beitrag „Die Gewalt der Sprachlosigkeit“:

„Dieses Sprechen folgt einem langen Schweigen - von Tätern, Betroffenen und anderen Beteiligten. Und das Schweigen ist nicht allein in einem Zusammenhang mit der Verdeckung schwerwiegender Straftaten zu sehen. Vielmehr verweist das Schweigen besonders auch auf die Form der sexuellen Gewalt, als eine im Nahbereich sich vollziehende tabuisierte Gewalt, die sich nicht im Physischen erschöpft, sowie - obwohl kaum thematisiert als eine auf gesellschaftliche Machtverhältnisse bezogene Gewalt, welche eine frühere Auseinandersetzung mit dem Thema erschwert oder sogar verhindert hat." (Thompson 2012, S. 118)

Ein Grund für die Sprachlosigkeit vieler Opfer, auf den Thompson hinweist, kann die Erfahrung sein, dass es ihnen mit ihrer Sprache nicht gelingt, Erwachsenen, denen sie sich mitteilen wollen, die Bedeutung der ihnen zugefügten sexuellen Gewalt nachvollziehbar zu machen. In dem Satz einer Frau, die viele Jahre ihrer Kindheit und Jugend in Heimen leben musste, kommt diese Erfahrung zum Ausdruck: „Ich konnte nicht länger schweigen - aber wer wird mir glauben?" (Kappeler 2014b; siehe auch Schünemann-Kroner 2014). Sie hatte auf der Veranstaltung des Jugendhilfeträgers, in dessen Heim sie Jahre ihrer Kindheit verbrachte, über die von ihr im Heimalltag erlebte Gewalt und die anhaltende traumatisierende Wirkung dieser Erfahrungen berichtet und war verzweifelt, als sie an den Reaktionen der ihr zuhörenden Professionellen merken musste, dass sie heute als Erwachsene ebenso wenig verstanden wurde wie damals als Kind und Jugendliche. Es ,wird nicht berücksichtigt, „,wie stark sexuelle Gewaltverhältnisse auf Alltagsräume des Sprechens und Handelns zurückgreifen können", schreibt Thompson (2012, S. 119). Das Sprechen und die Sprachlosigkeit seien an der Konstitution des Gewaltverhältnisses mittelbar und unmittelbar beteiligt. „Es ist die Sprachlosigkeit der Betroffenen, welche sexuellen Gewaltverhältnissen Dauer verleiht und eine Lösung der Betroffenen aus diesen erschwert" (ebd.). Die Erfahrung eines Kindes, die ihm zugefügte sexuelle Gewalt mit seinen sprachlichen und mimisch-gestischen Möglichkeiten Erwachsenen seines Bezugsfeldes mitteilen zu wollen, von diesen aber nicht verstanden oder nicht angehört zu werden, führt zu Verstummen, zu Sprachlosigkeit.

Über persönliche Erfahrungen sexueller Gewalt zu sprechen, ist für die meisten Betroffenen sehr schwer, selbst dann, wenn förderliche Bedingungen für dieses Sprechen wie Wertschätzung, Anerkennung, Diskretion, Verlässlichkeit gegeben sind. Immer muss eine schwere Verletzung der eigenen Integrität offengelegt werden, die die Gefahr einer Reaktivierung dieser Verletzung in sich birgt. Das ist ein weiterer Grund, warum es so wichtig ist, wie der Sprachraum beschaffen bzw. gestaltet ist, in dem die Gewalterfahrung ar- 
tikuliert werden kann, und welche Sprache dafür zur Verfügung steht. Dass für Kinder und Jugendliche Sprachraum und Artikulationsmöglichkeiten eine noch größere Bedeutung haben als für Erwachsene, muss, glaube ich, hier nicht weiter begründet werden. Christiane Thompson hat bezogen auf die Möglichkeiten von Kindern und Jugendlichen, die ihnen angetane sexuelle Gewalt artikulieren zu können, den emphatischen Begriff vom „Raum des Sagbaren“ geprägt (ebd., S. 124). Dieser Raum wird eingeengt und kann ganz verstellt werden, wenn im Heim, in der Kindertagesstätte, in anderen organisierten pädagogischen Settings Träger und Leitung von den Mitarbeitenden eine starke (kritiklose) Identifikation mit der Einrichtung verlangen. Daraus kann eine atmosphärische Geschlossenheit resultieren, deren Verletzung durch Einzelne moralisch und statusmäßig sanktioniert wird. Das betrifft die Kinder und Jugendlichen dann genauso wie die Mitarbeitenden, ja sogar Personen ihres sozialen Bezugssystems außerhalb der Einrichtung. In diesem Zusammenhang ist es wichtig, sich daran zu erinnern, das SprechenKönnen und Gehört-Werden ganz allgemein entscheidend davon abhängen, welchen Anteil ein Mensch am gesellschaftlichen Leben hat. Für die Gestaltung des Lebens von Kindern und Jugendlichen in pädagogischen Einrichtungen hat diese Erkenntnis eine besondere Bedeutung.

\section{Unterdrückung und Diskriminierung der Sexualtät von Kindern und Jugendlichen in pädagogischen Einrichtungen}

Über eine Variante sexueller Gewalt, die Unterdrückung und Diskriminierung der Sexualität von Kindern und Jugendlichen in pädagogischen Einrichtungen, soll hier abschließend noch geschrieben werden. Sie wird im gegenwärtigen Diskurs kaum thematisiert. Im Gegenteil: Seit den Aufdeckungen in katholischen Internatsschulen und der reformpädagogischen Odenwaldschule im Frühjahr 2010 ist es zu einem von konservativen Organisationen und Initiativen besorgniserregenden sexualmoralischen und sexualpädagogischen Rollback mit einem großen Medienecho gekommen, womit ein offener und wertschätzender Umgang mit Sexualität in pädagogischen Bezügen noch zusätzlich erschwert wird. Die Ausdrucksformen der Sexualität von Kindern und Jugendlichen werden in der institutionellen Erziehung oft genug verdrängt oder gar bewusst tabuisiert. Nicht selten werden ihre sexuellen Bedürfnisse, wenn sie von den Erziehenden nicht mehr ignoriert werden können, diskriminiert und sogar bekämpft. Diese Haltung und Praxis missachtet das sexuelle Selbstbestimmungsrecht, das Teil der Menschenwürde ist. Die Freiheit der sexuellen Selbstbestimmung gilt auch für Kinder und Jugendliche, die, wie Erwachsene, Träger von Menschen- und Grundrechten sind. Die UN-Kinderrechtskonvention spricht Minderjährigen ein besonderes Schutzrecht zu, weil ihre Rechte weltweit von Erwachsenen allzu oft missachtet und 
verletzt werden. In der IPPF-Erklärung über sexuelle Rechte heißt es, „dass die Entwicklung rechenschaftspflichtiger Strukturen unerlässlich ist, um die notwendigen Bedingungen für die Inanspruchnahme von Rechten zu schaffen. Solche Strukturen sollten nicht nur individuelle Abhilfe und Entschädigungsmaßnahmen vorsehen, sondern sie müssen sich auch mit den Macht-, Praxis- und Bedeutungsmechanismen, die zu Verletzungen sexueller Rechte führen können, auseinandersetzen und sie hinterfragen“(IPPF 2009).

Die Ablehnung der sexuellen Selbstbestimmung von Kindern und Jugendlichen durch eine sexualfeindliche Erziehung hat gravierende Folgen für die psycho-sexuelle Entwicklung. In Heimen, Internaten, Kinder- und Jugendgruppen wird oftmals eine pädagogische sexuelle Gewalt gegen alle Äußerungsformen des sexuellen Begehrens bzw. Luststrebens von Kindern und Jugendlichen jeder sexuellen Orientierung praktiziert. Das schon bei kleinen Kindern deutliche lustbetonte Interesse an den eigenen Geschlechtsorganen, ihre Neugier am anderen Geschlechtskörper, die Onaniepraktiken, erste heterosexuelle und ganz besonders gleichgeschlechtliche Zärtlichkeitsversuche, die Selbstaufklärung durch erotische Texte und Bilder, das ganze Spektrum der sexuell motivierten Neugier und der ihm zugehörenden Handlungen wird mit einer moralisierenden und diskriminierenden Sprache der Erziehenden entwertet. Die von einer solchen sexualfeindlichen Erziehung betroffenen Kinder und Jugendlichen haben kaum eine Chance, sich eine eigene, nicht diskriminierende, die sexuellen Lust- und Zärtlichkeitsaspekte wertschätzende Sprache für ihr autoerotisches Selbstgespräch und für die Kommunikation mit Gleichaltrigen und Erwachsenen anzueignen. Dazu brauchen Kinder sprachliche „Vorbilder“, die sie im „Sprachraum“ einer sexualfeindlichen Erziehung nicht finden können. Mit dem systematischen Verweis der Sexualität, genauer der Formen des sexuellen Begehrens, in dem sie sich äußert, in den Bereich des Unmoralischen und Ungehörigen, wird von klein auf ein Schuldbewusstsein in den Heranwachsenden ausgebildet, das sich schließlich nicht nur auf Handlungen, sondern auch auf ,unkeusche Gedanken“ bezieht, auf die jedem Menschen eigenen sexuellen Phantasien.

Über die hier skizzierte sexualfeindliche Erziehungspraxis, die bis zu regelrechten Verfolgungen und handfesten Strafen reicht (körperliche Züchtigungen und Bloßstellungen wegen Onanierens, minutiöse Kontrollpraktiken, die den Intimbereich der Kinder und Jugendlichen missachten), wurde und wird von fast allen ehemaligen Heimkindern berichtet, die Zeiten ihrer Kindheit und Jugend in kirchlichen und anderen Erziehungseinrichtungen verbringen mussten. Auch in den jüngsten Berichten der Opfer sexueller Gewalt in katholischen Internatsschulen wird auf diese Praxis als Teil der erfahrenen sexuellen Gewalt hingewiesen, ohne sie so zu benennen.

Dass die sexualmoralische Prägung von Erzieherinnen und Erziehern sich auf ihren Umgang mit Kindern und Jugendlichen unmittelbar auswirkt, ist eine bekannte Tatsache. Ganz allgemein gilt, dass Erzieherinnen und Erzieher 
mit einer von Schuldgefühlen belasteten Sexualität, die sie immer wieder in Gewissenskonflikte mit dem eigenen sexuellen Begehren bringt, auf die Sexualität der Heranwachsenden, die sie erziehen sollen, mit einer schwierigen Mischung aus Abwehr und Verlangen reagieren. So ist es für sie zum Beispiel schwer, erzieherisch angemessen zu handeln, wenn ein Kind, mehr noch ein Jugendlicher oder eine Jugendliche, sich in sie verliebt oder auch nur in dem Bedürfnis nach ein bisschen Zärtlichkeit ihre körperliche Nähe sucht. Solche Wünsche/Bedürfnisse von Kindern/Jugendlichen sind normal und legitim. Bei Kindern/Jugendlichen, die in Internatsschulen oder Heimen der Jugendhilfe aufwachsen müssen, können sie aufgrund der emotionalen Entbehrungen und Belastungen, die sie aus ihren Herkunftsfamilien oft mitbringen, besonders heftig und fordernd werden. Es gehört zu den wichtigsten Fähigkeiten von Pädagoginnen und Pädagogen, damit so umgehen zu können, dass diese Kinder/Jugendlichen nicht noch weiter belastet werden und eine Chance bekommen (Pubertät als ,zweite Chance“), ihre negativen Kindheitserfahrungen neu zu ,sortieren“ und besser verarbeiten zu können. Von Erzieherinnen sowie Erziehern und auch von Lehrerinnen sowie Lehrern mit einer restriktiven abwehrenden Sexualmoral werden die von Kindern/Jugendlichen an sie gerichteten Bedürfnisse/Wünsche leicht als Versuchung erlebt, die sie brüsk zurückweisen müssen, um sich selbst zu schützen. Tatsächlich kann es sich auch um eine reale „Versuchung“ handeln, wenn pubertierende Jugendliche sich in für sie attraktive Erzieherinnen oder Erzieher ernsthaft und schwärmerisch verlieben. In Schulen, Internaten und Heimen, aber auch in der offenen Jugendarbeit kommt das nicht selten vor; vor allem dann, wenn die Jugendlichen in der pädagogischen Einrichtung wenig Gelegenheit und Freiheit haben, altersgemäße Kontakte zu knüpfen und ihre eigene erotische Attraktivität zu testen. Solche erotischen Annäherungsversuche von Heranwachsenden an die sie erziehenden Erwachsenen gehören zu ihrem sexuellen Selbstbestimmungsrecht, dem seine Grenze durch die Erwachsenen gesetzt werden muss, indem sie das sexuelle Begehren des/der Jugendlichen, ohne es zu diskriminieren, unzweideutig zurückweisen.

Es gehört zur Professionalität pädagogischer, psychologischer und medizinischer Fachkräfte, diese Grenze ziehen zu können, ohne das Kind/den Jugendlichen zu kränken, seine Gefühle zu entwerten und es/ihn zu demütigen. Um das zu können, benötigen die Erziehenden eine Sprache, mit der sie dem Mädchen/dem Jungen vermitteln können: „Aus meiner Verantwortung für Dich, für Deine Integrität und Entwicklung, aber auch aus Verantwortung für mich selbst, darf und kann ich Dich nicht lieben in dem Sinne, wie Du Dir das wünschst. Aber Du interessierst mich und ich mag Dich.“ 


\section{Resümee}

Die bewusste Anerkennung der Tatsache, dass alle sich in einer pädagogischen Einrichtung begegnenden Menschen auch sexuelle Wesen sind und die Aufgabe haben, eine möglichst befriedigende, andere nicht beeinträchtigende oder gar schädigende sexuelle Praxis zu entwickeln, schließt ein, Sexualität nicht primär unter dem Aspekt von drohenden Gefahren und Gefährdungen $\mathrm{zu}$ betrachten, denen präventiv begegnet werden müsse. Eine aufgeklärte Sexualerziehung versteht Sexualität als eine das Leben bereichernde anthropologische Grundtatsache, die es zu entfalten und gegen alle politischen, ideologischen und kommerziellen Funktionalisierungen zu behaupten gilt. Mechthild Wolff schreibt dazu:

„Schutz und Sicherheit sind auch eine wichtige Grundbedingung für die Ausbildung einer sexuellen Identität als Teil der Ich-Identität. Dies impliziert, dass Kinder befähigt sind, den eigenen Körper und die Körper anderer Menschen zu entdecken, eigene sexuelle Bedürfnisse und die anderer Personen wahrzunehmen, einzuordnen und altersadäquat damit umgehen zu können. [...] Dies alles setzt die Ausbildung eigener Schutzbedürfnisse voraus, ein sensibles Körper- und Lustempfinden und eine gegenseitige Bedürfnisartikulation. Damit Kinder und Jugendliche Liebesfähigkeit ausbilden, benötigen sie eine Umgebung, in der sie geschützt darüber reden, sich darüber austauschen und mit anderen kritisch reflektieren können. Werden diese Themen tabuisiert oder wird Kindern kein Entwicklungsschonraum eingeräumt, der frei von Gewalt und Machtmissbrauch ist, können sie auch keine selbstbestimmte sexuelle Identität ausprägen.“(Wolff 2015, S. 680)

Manfred Kappeler, Prof. Dr., war von 1989 bis 2005 Hochschullehrer für Erziehungswissenschaften/Sozialpädagogik an der Technischen Universität Berlin und ist seit 25 Jahren in der Heimerziehung, offenen Jugendarbeit, Drogenarbeit, Supervision, Fort- und Weiterbildung sozialpädagogischer Fachkräfte tätig.

\section{Literatur}

Böhler, Dietrich/Gronke, Horst (2011): Stichwort „Diskurs“. In: Kolmer, P./ Wildfeuer, A. G. (Hrsg.): Neues Handbuch philosophische Grundbegriffe. Band 1. Freiburg: Verlag Karl Alber, S. 559.

Böllert, Karin/Wazlawik, Martin (Hrsg.) (2014): Sexualisierte Gewalt. Institutionelle und professionelle Herausforderungen. Wiesbaden: Springer VS.

International Planned Parenthood Federation (IPPF) (2009): Sexuelle Gewalt: Eine IPPF-Erklärung. https:/www.profamilia.de/fileadmin/publikationen/profa milia/IPPF_Deklaration_Sexuelle_Rechte-dt2.pdf [Zugriff: 3. April 2017]. 
Kappeler, Manfred (2011): Anvertraut und Ausgeliefert. Sexuelle Gewalt in pädagogischen Einrichtungen. Berlin: Nicolaische Verlagsbuchhandlung.

Kappeler, Manfred (2014a): Anvertraut und Ausgeliefert - Sexuelle Gewalt in pädagogischen Einrichtungen. In: Böllert, K./Wazlawik, M. (Hrsg.): Sexualisierte Gewalt. Institutionelle und professionelle Herausforderungen. Wiesbaden: Springer VS, S. 7-19.

Kappeler, Manfred (2014b): „Ich konnte nicht länger schweigen - aber wer wird mir glauben?" Über die Traumatisierungen ehemaliger Heimkinder. In: Widersprüche. Zeitschrift für sozialistische Politik im Bildungs-, Gesundheits- und Sozialbereich 34, 131, S. 9-20.

Kappeler, Manfred (2015): Betroffenenorganisationen und BetroffenenvertreterInnen im Spannungsfeld der Aushandlung an den Runden Tischen. In: Fegert, J. M./Wolff, M. (Hrsg.): Kompendium „Sexueller Missbrauch in Institutionen“. Entstehungsbedingungen, Prävention, Intervention. Weinheim/Basel: Beltz, S. 83-103.

Schünemann-Kroner, Annelen (2014): Heimkinder sollen ihre Vergangenheit vergessen. In: Widersprüche. Zeitschrift für sozialistische Politik im Bildungs-, Gesundheits- und Sozialbereich 34, 131, S. 33-37.

Thompson, Christiane (2012): Die Gewalt der Sprachlosigkeit. In: Thole, W. et al. (Hrsg.): Sexualisierte Gewalt, Macht und Pädagogik. Opladen/Berlin/Toronto: Verlag Barbara Budrich, S. 118-128.

Wolff, Mechthild (2015): Schutz und Sicherheit als Entwicklungsvoraussetzungen für das Aufwachsen von Kindern und Jugendlichen. In: Fegert, J. M./Wolff, M. (Hrsg.): Kompendium ,Sexueller Missbrauch in Institutionen“". Entstehungsbedingungen, Prävention, Intervention. Weinheim/Basel: Beltz, S. 673-682. 